\title{
Evaluation of the SR based auto-tuning image enhancement system
}

\author{
Noriko Kojimaa, ${ }^{\mathrm{a},}$, Bikash Lamsal ${ }^{\mathrm{b}}$, Naofumi Matsumoto ${ }^{\mathrm{a}}$, Mitsuo Yamashiro ${ }^{\mathrm{a}}$ \\ aAshikaga University, 268-1, Omae-cho, Ashikaga, Tochigi 326-8558, Japan. \\ b KAJIMA Corporation, Technical Research Institute2-19-1, Tobitakyu, Chofu, Tokyo 182-0036, Japan \\ *Corresponding Author: n.kojima0904@gmail.com
}

\begin{abstract}
This paper proposes the evaluation of an auto-tuning stochastic resonance (SR) for image enhancement on images under various illumination conditions. The current process is developed being based on our previous works related to the image enhancement by using the manual tuning stochastic resonance. The process was performed by adding the random noise and threshold 0 in an image. The process works properly in the dark and very low contrast images as well as bright images. This image enhancement system works on dark and bright images as well. The system was tested with the face detection algorithm on the dark and illumination variant images. In this paper, we present the idea of auto-tuning of the SR iteration with random noise and threshold value 0 by using the process related to the histogram calculation, mean and median. In this paper, we performed various experiments on object and human detection as well under different conditions and confirmed the effectiveness of our auto-tuning SR based image enhancement algorithm.
\end{abstract}

Keywords: image enhancement, stochastic resonance, parameter auto-tuning, perceptual quality metric

\section{Introduction}

In an image obtained from a consumer based digital camera, it is very difficult to discriminate an object to be detected from an image due to the several shooting situations under various illumination conditions such as lightening, back lightening, darkness, brightness etc. To detect the presence of the proper object from such type of image is a difficult task. To overcome such type of problems, contrast adjustment process is being used from several years. This is a process of expanding the width of the histogram and converting the density for an image which is difficult for vision due to the absence of the difference in the brightness of the image.

But, there exists a lot of case images where the image cannot be enhanced by the contrast adjustment process, which has been clarified in our previous researches related to the face and human body detection on dark and illumination variant images ${ }^{(1,2,3)}$. The another process for image enhancement on such type of images is image sharpening and filtering, that enhances high frequency components of edges and details for optically degraded images. But, this process focus on the contrast adjustment process described before.

The use of stochastic resonance (SR) as an idea of enhancing an image with strict lighting conditions and clear the detection target is shown in the various researches ${ }^{(4,5,6)}$. But, all of these researches do not focus on dark and illumination variant images. Also, doesn't deal with the auto-tuning parameters in SR. Therefore, there remains a margin for considering the image enhancement algorithm based on SR.

In our previous researches, we proposed an image enhancement algorithm based on SR ${ }^{(1,2,3)}$. The SR parameters were tuned manually in our previous works. Upgrading our previous researches, we are proposing an image enhancement method that auto tunes the SR parameters in order to solve the difficult problem of feature detection and recognition due to the influence of contrast such as illumination variant, dark and bright image.

Our current method of auto-tuning SR is tested by using the dark and illumination variant images used in various fields. Once the image is enhanced, it is then combined with face detector or human detector for detecting the presence of face or human in the given image. Our previous works were focused on the face detection, so the images were experimented by combining with the face detector. 
In the current research, we are experimenting our method for human detection as well. The current system can be applied in the automatic car for human detection in the night time or dark environment. We are using the Open $\mathrm{CV}$ based human detector for the experimentation. The enhanced images are also compared with the contrast adjustment process for clarifying the effectiveness of our proposed method.

\section{Structure of the Proposed method}

The proposed method is structured in various steps. The stochastic resonance (SR) process is being implemented and modified for the image enhancement on dark and illumination variant images under strict shooting situations. The basic SR algorithm along with our modified process for image enhancement are briefly described below.

\subsection{Basic Principle of SR}

The basic principle of SR was developed by Benzi ${ }^{(7)}$. The process of the basic SR is shown in figure 1. SR was first developed to work on the field of the signal processing, but we have applied this process in the field of image processing for image enhancement as shown in figure 2 .

In the figure 2, noisy and unclear image is made clear by applying the stochastic resonance technique. The noise and threshold is applied in a proper amount for getting the proper result. The noise value and the threshold value is manually tuned in this process.

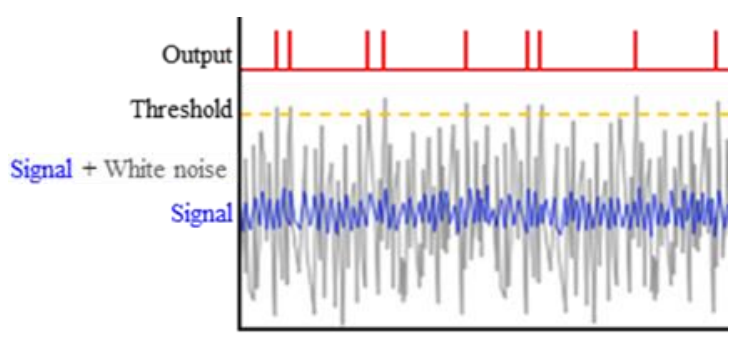

Fig.1. Basic principle of stochastic resonance.

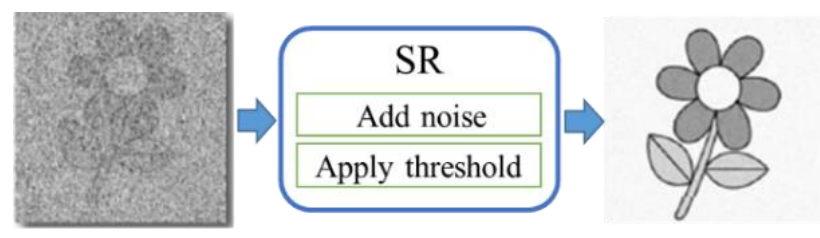

Fig.2. Applying SR idea for image enhancement

\subsection{Collins type SR}

This technique was implemented by Collins et.al. ${ }^{(8)}$ for the time series signal processing as shown in figure 3 . We adopted this idea for the image enhancement by following the Pulse density modulation ${ }^{(9)}$ in our process. This method is a modification of the original SR to parallel summation processing as shown in figure 4, where the tuning of the $n\left(N_{\text {tune }}\right)$ is required. The $N$ is tuned manually in this process by checking the enhanced level of the image.

In figure 5, the preliminary experiment was carried out by using the noise level at 3 and threshold value 0 . In the original image, nothing is seen clearly, as the $N_{\text {tune }}$ level increases, the people in the image along with the persons in the background are confirmed. From the histogram, we can see that the histogram spreads in a bright direction as $N_{\text {tune }}$ increases. Depending on the situation of the original image, when $N_{\text {tune }}$ reaches a certain value, face detection of the person in front is possible. In this image, the face is detected at the value of $N_{\text {tune }}=18$.

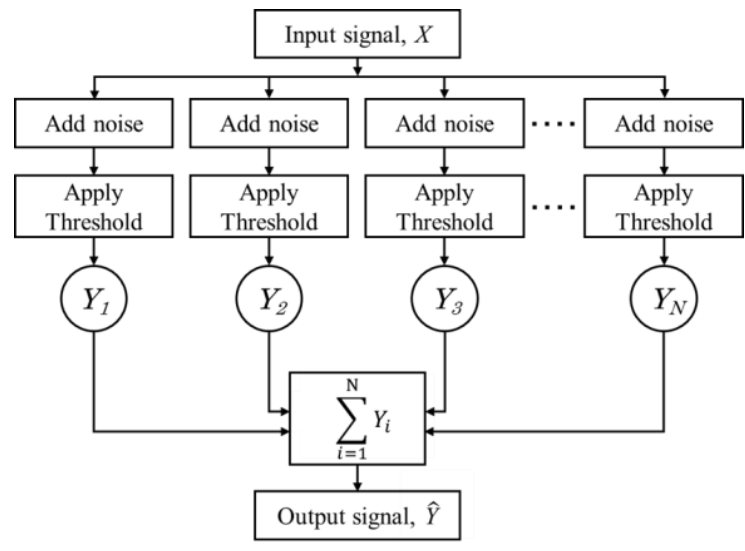

Fig.3. Flow chart of the Colins type SR fro signal processing

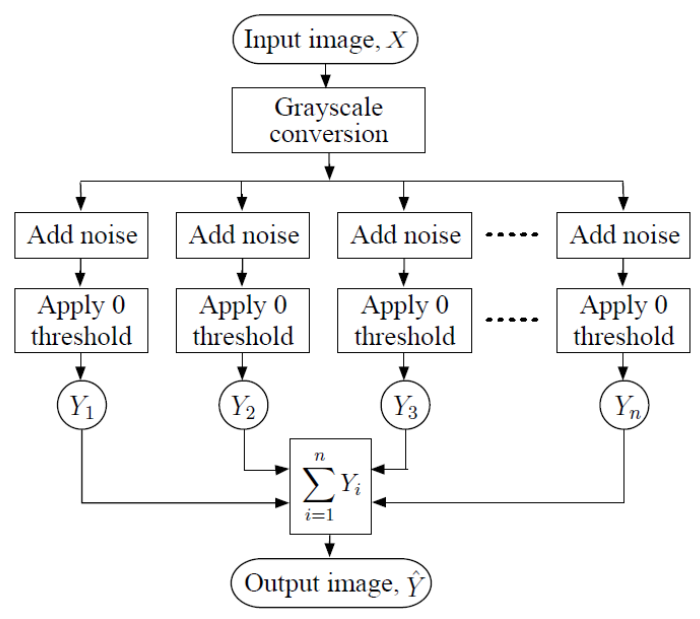

Fig.4. Flow chart of the Colins type SR for image enhancement 

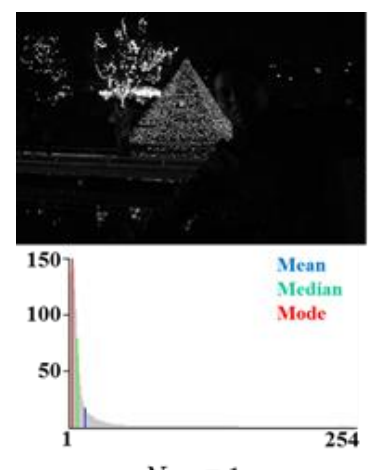

$N_{\text {tune }}=1$
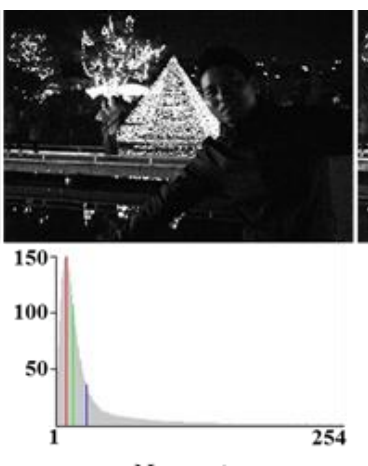

$N_{\text {tune }}=4$
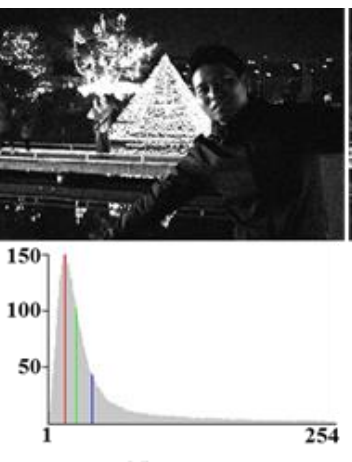

$N_{\text {tune }}=7$
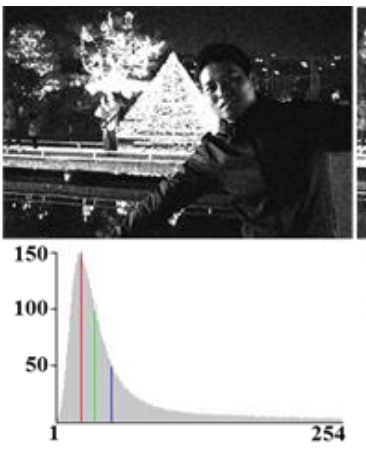

$N_{\text {tune }}=10$
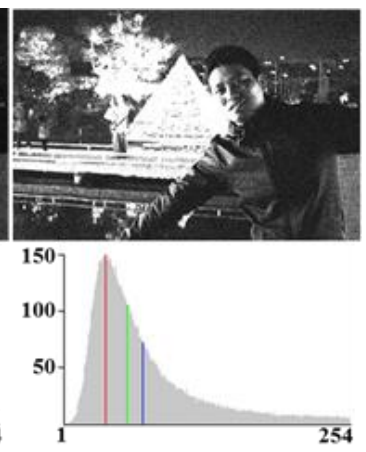

$N_{\text {tune }}=18$

Mean $>60 \cdot \alpha$

Median $>50 \cdot \alpha$

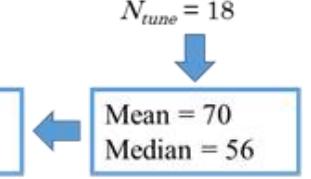

Fig.5. Effect of the iteration number of Collins type SR; $N_{\text {tune }}$.

\subsection{Proposed SR algorithm}

The proposed SR algorithm is the upgraded system of Collins type SR, where the SR parameters are automatically tuned instead of manual tuning. We have introduced the $N_{\text {tune }}$ auto-tuning mechanism in our proposed method. The flow of the proposed algorithm is shown in figure 6 .

According to the flow diagram shown in figure $6, \mathrm{X}$ is the original image, $\mathrm{Y}$ is the image corrected or enhanced by using the SR technique, $Y_{i}$ represents the $i_{\text {th }}$ number of the SR process and $\alpha$ represents the safety factor for detection and set to 1.1. As described above, the intensity of the white noise was 3 and the threshold value was 0 from the result of the preliminary experiment.

By setting the threshold value to 0 , occurrence of a negative gradation value generated by the random noise can be excluded. This prevents from diminishing the important information in the dark images. Also, from the preliminary experiment, we set the conditional expression to repeat until the Mean $>60 \cdot \alpha$ and Median $>50 \cdot \alpha$.

In the current process, the range of the histogram is set from 1-254 instead of 0-255 because 0 and 255 shows the final black level and final white level of the image which makes the calculation of the mean, median and mode a little complicated.

\section{Experimentation and Evaluation of the Proposed SR algorithm}

\subsection{Preparation for experiments}

An auto-tuning system was developed based on the algorithm shown in figure 6 . In this system, mean and median are obtained after stochastic resonance processing. If mean and median satisfy the condition, the image enhancement process by using SR gives an output enhanced image. The safety factor $\alpha$ for mean and median was set to 1.1 for preventing the conditions to be satisfied before the required output image due to the presence of many image pixels at 0 or 255 .

We have developed a $\mathrm{C}$ programming code by using the Visual studio 2010 and the Open CV library on PC (i7-5820) using Windows 8.164 bit 0.S.

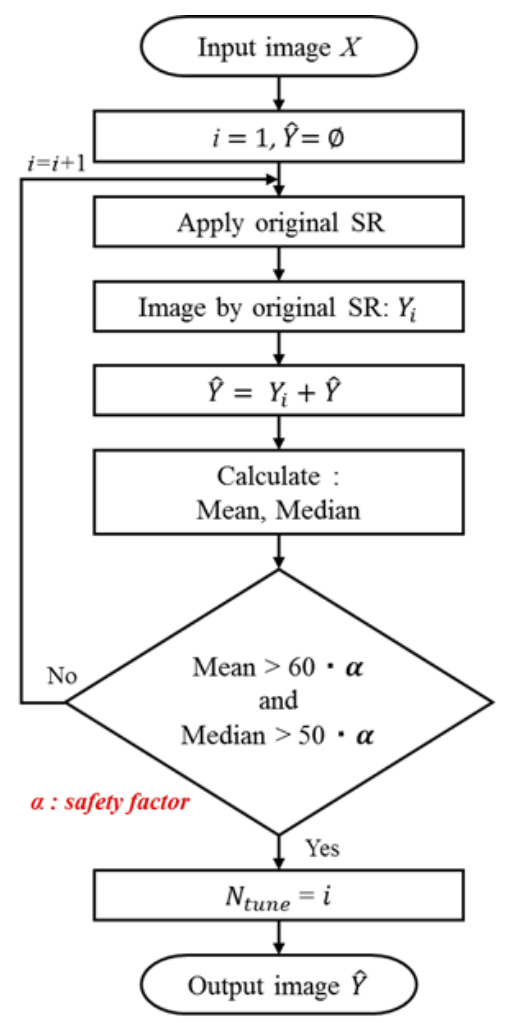

Fig.6. Proposed SR algorithm for image enhancement 


\subsection{Experimental results and evaluation}

We evaluated our proposed SR algorithm by experimenting the various images along with the human detector and face detector. We used the high performance face detector proposed by Bikash et.al, ${ }^{(10)}$ and Open CV ${ }^{(11)}$ human detector for detecting the presence of the human in the input image.

We also, used Perceptual Quality Metric (PQM) of images for evaluation of our proposed method. PQM is a metric that quantifies the image quality from 1 to 10 grades, the $10^{\text {th }}$ grade is evaluated as high quality. Our proposed method is then compared with the image enhanced by using the contrast adjustment process.

In this paper, we present the 3 different image cases, including the experimental results and the histograms along with the PQM as shown in figure 7. The comparison of the histogram and the images obtained from the contrast adjustment process with the output of our proposed method is shown along with the result of human and face detection. The case A image was taken in our laboratory by switching off the lights and made the rom dark. In the image, several PC displays are in the background. In this case, when $N_{\text {tune }}$ $=9$, the mean and median satisfied the image enhancement condition and the human detector along with the face detector was applied. The output result of our proposed SR detects both the human in the red box and face in the yellow box. In case of the result of the contrast adjustment process, only the human is detected, but not the face. The execution time is $435.8 \mathrm{~ms}$. The value of PQM is close to 10 when $\mathrm{N}=6$, but the face is detected with $\mathrm{N}=9$, exceeding the value.
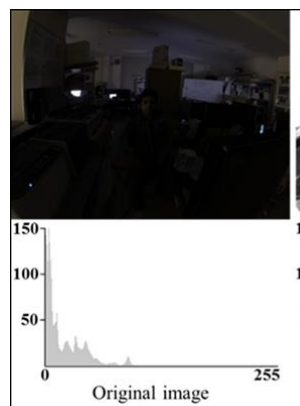

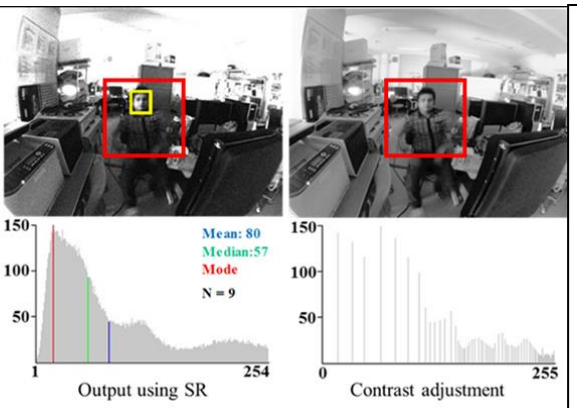

Case A

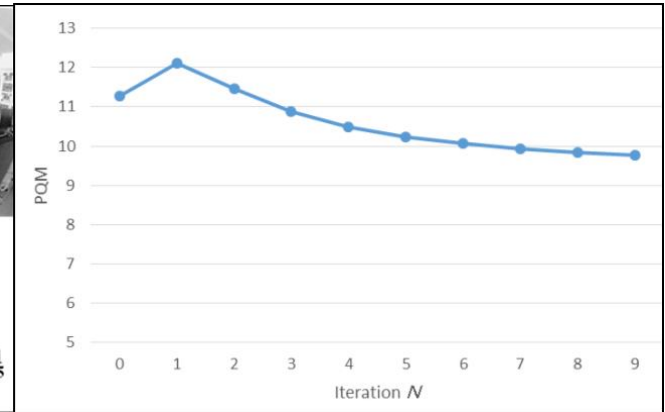

Iteration $N$
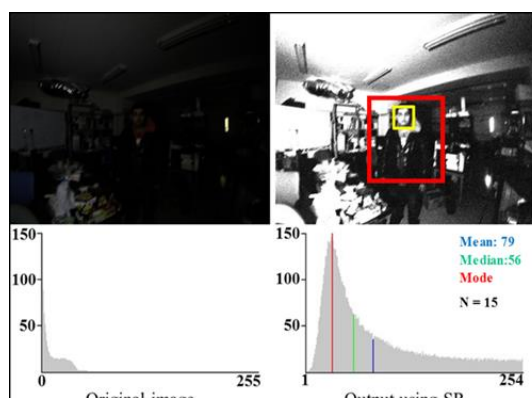

Output using SR
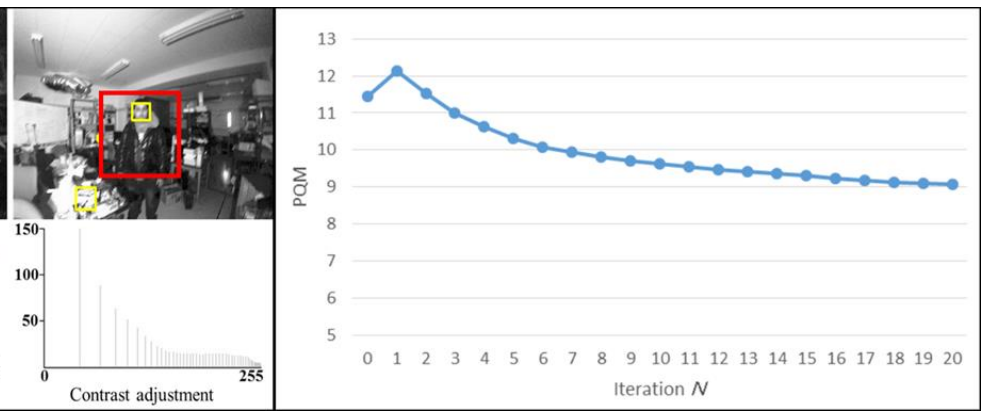

Case B
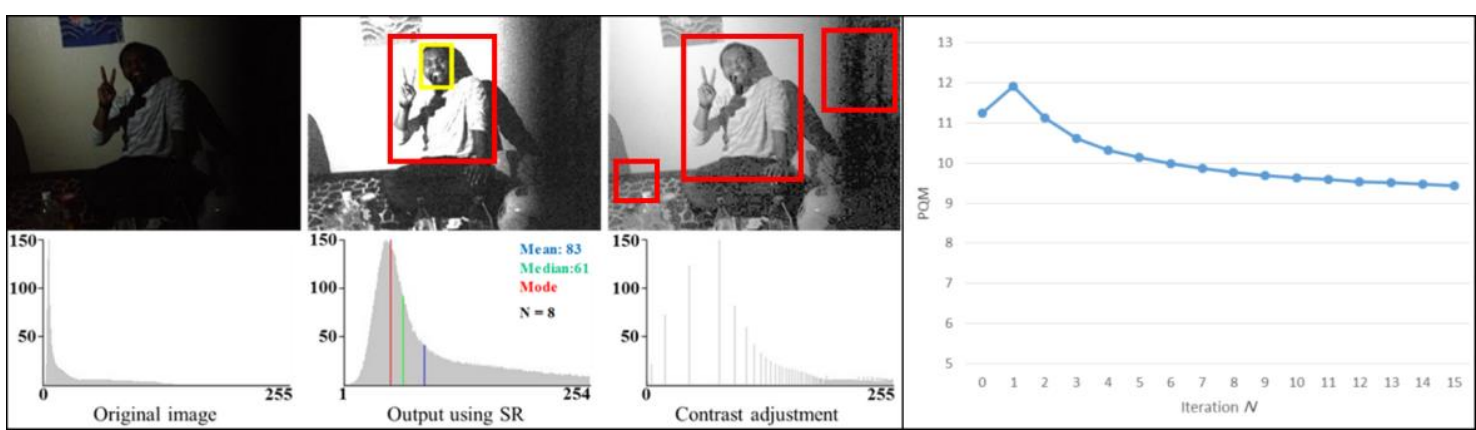

Case C

Fig.7. Experimental results of our Proposed SR algorithm 
The case B is an image taken in the laboratory without any lightings. In this case, when $N_{\text {tune }}=15$, the mean and median satisfied the image enhancement condition and the human detector along with the face detector was applied. In this case, both the face and human body is detected. Even the face and the human body is detected on the contrast adjustment image as well but, the fine error occurred at a position other than the face. The value of PQM is close to 10 when $\mathrm{N}=6-7$, but the face is detected with $\mathrm{N}=15$. The execution time is $441.9 \mathrm{~ms}$.

The Case $\mathrm{C}$ is an image of a person with dark skin color, taken in a dark environment without flash. In this case, when $N_{\text {tune }}=8$, the mean and median satisfied the image enhancement condition and the human detector along with the face detector was applied. In this case, both the face and human body is detected. Even the face and the human body is detected on the contrast adjustment image as well but, the fine error occurred at a position other than the face. The value of PQM is close to 10 when $\mathrm{N}=5$, but the face is detected with $\mathrm{N}=8$. The execution time for this case is $688.62 \mathrm{~ms}$.

In the cases $\mathrm{A}-\mathrm{C}$, we can see from the histogram of the contrast adjustment method that the entire image tends to be somewhat blurred. It is because, the gradation value diminishes in this method due to which the face is not detected in some cases. Our proposed method enhances the shadow of the image, due to which the difference in the brightness of the face to be detected became clear and the face was detected accurately.

\subsection{Comparison with Contrast adjustment process}

In order to validity the effectiveness of our proposed system, we compared and tested our proposed system with the contrast adjustment process under various difficult illumination conditions.

As shown in figure 7 , case $\mathrm{B}$, the human in the image along with the face is detected by using our proposed algorithm as well as by the contrast adjustment process. If we look clearly at the contrasted adjusted images after applying the detector, we can see the detected objects rather than the face and the human, which can be regarded as error. It is because, the contrast adjustment method is a process of expanding the width of the histogram and converting the density. As we can see the histograms shown in figure 8 is the result of non-continuous performance of the interpolation between the gradations values are not performed continuously.

But our proposed method the interpolation between the gradations values are performed continuously, which results in the proper histogram and clearly enhanced images. Since face detector and human detector are a method of detecting a feature pattern of the face and the human body from the contrast difference, we think that our proposed method is effective.

\section{Discussion and Conclusion}

We proposed an image enhancement method using stochastic resonance (SR), which auto tunes the parameters related to the SR and clarified the effectiveness of the proposed method by the experiments. Unlike from the contrast adjustment process, the histogram is not only extended but are interpolated as well. Since the histogram is interpolated, the contrast difference was enhanced.

Based on the face detection and human detection experimentation, the proposed method showed a possibility of reducing the detection error in compared to the contrast adjustment process. In case of the human detection, it was necessary to measure the degree of image enhancement by using the PQM. From the experiment performed for comparing the face detection and PQM evaluation value, we found a lot of cases, where the face detection and human detection was not satisfied even when the PQM value was near to 10 .

The auto-tuning SR based image enhancement was developed to work on the illumination variant images. The system can be applicable in the various fields that uses image. The proposed system will help to increase the effectivity of the image processing system that depends on the image enhancement. Our next applicable images for research will be related to the medical images.

\section{References}

(1) B. Lamsal, N. Kojima, and N. Matsumoto: "Impact of the stochastic resonance on dark and illumination variant images for face detection", Journal of the Institute of Industrial Applications Engineers. vol. 3, no. 4, pp. 167-173, (2015)

(2) N. Kojima, B. Lamsal, N. Matsumoto: “An adaptive tuning stochastic resonance approach for image enhancement on illumination variant images" , Journal of the Institute of Industrial Applications Engineers vol.5, no.1, pp.1-8, (2017)

(3) N. Kojima, N. Matsumoto and M. Yamashiro: "Image enhancement under severe lighting conditions using 
stochastic resonance” , LIFE2017, (2017) (in Japanese)

(4) E. Simonotto, M. Riani, S. Charles, M. Roberts, J. Twitty and F. Moss: "Visual perception of stochastic resonance", Phys. Rev. Lett., 78(6): pp.1186-1189, (1997)

(5) R. Chouhan, R. K. Iha and P. K. Biswas: "Wavelet-based contrast enhancement of dark images using dynamic stochastic resonance” , ICVGIP' 12, Mumbai, India, (2012)

(6) R. K. Jha and R. Chouhan: "Noise-induced contrast enhancement using stochastic resonance on singular values” , SIViP 8:pp.339-347, (2014)

(7) R. Benzi, A. Sutera and A. Vulpiani: "The mechanism of Stochastic Resonance", J.Physics. A: Math and General, vol.14, pp. L453 -L457,

(8) J. J. Collins, C. C. Chow and T. T. Imhoff: "Stochastic resonance without tuning", Letters to NATURE, vol. 376, pp. 236-238, (1995)

(9) S. Kasai, K. Miura and Y. Shiratori, "Threshold-variation enhanced adaptability of response in a nanowire field-effect transistor network", Applied Physics Letters, Vol.96, No.19, pp.194102, 2010. DOI:10.1063/1.3428784

(10)B. Lamsal and N. Matsumoto, "Effects of the Unscented Kalman filter process for high performance face detector", Intl. J. of Information and Electronics Engineering, Vol.5, No.6, pp.454-459, 2015. DOI: 10.7763/IJIEE.2015.V5.578.

(11) http://opencv.jp/ 